\section{(1)}

CrossMark

\title{
Morphomolecular motifs of pulmonary neoangiogenesis in interstitial lung diseases
}

\author{
Maximilian Ackermann (10 1,2,10, Helge Stark $\mathbb{1}^{3,10}$, Lavinia Neubert ${ }^{3,4}$, \\ Stephanie Schubert ${ }^{3}$, Paul Borchert ${ }^{3}$, Friedemann Linz ${ }^{1}$, Willi L. Wagner ${ }^{5,6}$, \\ Wolfram Stiller ${ }^{5,6}$, Mark Wielpütz $^{5,6}$, Anne Hoefer ${ }^{3}$, Axel Haverich ${ }^{4,7}$, \\ Steven J. Mentzer ${ }^{8}$, Harshit R. Shah ${ }^{3,4}$, Tobias Welte $\mathbb{1}^{4,9}$, Mark Kuehnel ${ }^{3,4,10}$ \\ and Danny Jonigk ${ }^{3,4,10}$
}

@ERSpublications

Pulmonary injury patterns are the common denominator in progression of fibrosing lung diseases. This study identified distinct manifestations of neoangiogenesis as drivers of pulmonary remodelling in human ILDs, modulated by bone marrow-derived monocytes. http://bit.ly/34F7KNS

Cite this article as: Ackermann M, Stark H, Neubert L, et al. Morphomolecular motifs of pulmonary neoangiogenesis in interstitial lung diseases. Eur Respir J 2020; 55: 1900933 [https://doi.org/10.1183/ 13993003.00933-2019].

ABSTRACT The pathogenetic role of angiogenesis in interstitial lung diseases (ILDs) is controversial. This study represents the first investigation of the spatial complexity and molecular motifs of microvascular architecture in important subsets of human ILD. The aim of our study was to identify specific variants of neoangiogenesis in three common pulmonary injury patterns in human ILD.

We performed comprehensive and compartment-specific analysis of 24 human lung explants with usual intersitial pneumonia (UIP), nonspecific interstitial pneumonia (NSIP) and alveolar fibroelastosis (AFE) using histopathology, microvascular corrosion casting, micro-comupted tomography based volumetry and gene expression analysis using Nanostring as well as immunohistochemistry to assess remodellingassociated angiogenesis.

Morphometrical assessment of vessel diameters and intervascular distances showed significant differences in neoangiogenesis in characteristically remodelled areas of UIP, NSIP and AFE lungs. Likewise, gene expression analysis revealed distinct and specific angiogenic profiles in UIP, NSIP and AFE lungs.

Whereas UIP lungs showed a higher density of upstream vascularity and lower density in perifocal blood vessels, NSIP and AFE lungs revealed densely packed alveolar septal blood vessels. Vascular remodelling in NSIP and AFE is characterised by a prominent intussusceptive neoangiogenesis, in contrast to UIP, in which sprouting of new vessels into the fibrotic areas is characteristic. The molecular analyses of the gene expression provide a foundation for understanding these fundamental differences between AFE and UIP and give insight into the cellular functions involved. 


\section{Introduction}

The term interstitial lung disease (ILD) refers to a complex group of hundreds of non-neoplastic pulmonary diseases with variable morphologies, clinical presentations and progression tendencies. ILD is characterised by an aberrant response to injury, generally leading to compartment-specific disruptions of lung architecture [1]. In wound healing, the impact of angiogenesis and physiological vascular architecture has long been recognised as a crucial element, particularly in the interaction of granulation tissue and extracellular matrix [2]. Although blood vessels make up $>70 \%$ of normal lung parenchyma volume $[3,4]$, the influence of pulmonary microvascular changes on fibrotic remodelling in humans and especially on the progress of ILD remains poorly understood.

The pivotal role of neoangiogenesis in idiopathic pulmonary fibrosis (IPF), an aggressive variant of the idiopathic interstitial pneumonias, was revealed by EBINA et al. [5], who could demonstrate an increased capillary density with increased endothelial proliferation in areas of minimal fibrosis, but decreased density in the adjacent, most extensively remodelled fibrotic lesions in afflicted patients.

Microvascular corrosion casting and high-resolution computed tomography (micro-computed tomography) are well suited to visualise and analyse the vascular structure of lung tissue [6]. Using these methods, the heterogeneous complexity of the vasculature in neoplasias was characterised [7-9]. Here, highly vascularised, capsule-like structures around (myo)fibroblastic foci [10] were described. In ILD, the only previous demonstration of prominent vascular obstruction was in lungs of patients with IPF [11].

Aberrant expression of a multitude of angiogenesis-related molecular mediators has been described in the lungs and blood of IPF patients, including vascular endothelial growth factor (VEGF), basic fibroblast growth factor, CXC chemokines with ELR motif and transforming growth factor (TGF)- $\beta$. In addition, the highly angiostatic molecule pigment epithelium-derived factor was found to be overexpressed within the fibroblastic foci, typical (but not specific) for the usual interstitial pneumonia (UIP) pattern commonly seen in IPF $[10,12]$. In a bleomycin-induced murine fibrosis model, CXCL11 reduced pulmonary fibrosis and the absolute number of endothelial cells found in the remodelled compartments [13]. Our own studies on the antiangiogenic multikinase inhibitor nintedanib [14, 15] revealed an attenuation of aberrant microvascular architecture. In detailed studies, we and others found an overall decreased vascular density with normalisation of vessel diameters [16] and a reduction of CCL18 secretion and macrophage polarisation in treated lungs $[17,18]$.

In a computed-tomography analysis of the degrees of fibrotic remodelling in IPF patients, JACOB et al. [19] reported on the impact of radiological variables for predicting progression and mortality. They demonstrated that pulmonary vascular volumes had the strongest correlation with mortality [19]. We and others have repeatedly hypothesised that microvascular alterations represent (one of) the first step(s) in the pathogenesis of IPF [20]. The loss of vascular integrity and the destruction of the alveolar-capillary membrane drives the continuous remodelling of the pulmonary architecture by pro-angiogenic and pro-fibrotic stimuli [16]. Recent observations also demonstrated that pulmonary capillaries can undergo an endothelial-mesenchymal transition in bleomycin-induced fibrosis [21].

Given the significant role of angiogenesis in repair reactions, one can reasonably assume that varying microvascular alterations may be reflections of both the differing response-to-injury patterns and the subsequent remodelling of the pulmonary architecture observed in ILD [22, 23]. Prior studies have focused predominantly on epithelial-mesenchymal pathogenesis of ILD, while possible vascular mechanisms remain poorly characterised or understood. Therefore, the aim of our present study was to characterise the morphological and molecular differences of vascularisation and angiogenesis in ILD, focusing on the

Affiliations: ${ }^{1}$ Institute of Functional and Clinical Anatomy, University Medical Center of the Johannes Gutenberg-University Mainz, Mainz, Germany. ${ }^{2}$ Institute of Pathology, Medical Faculty, Heinrich-HeineUniversity and University Hospital Düsseldorf, Düsseldorf, Germany. ${ }^{3}$ Institute of Pathology, Hannover Medical School, Hannover, Germany. ${ }^{4}$ Member of the German Center for Lung Research (DZL), Biomedical Research in Endstage and Obstructive Lung Disease Hannover (BREATH), Hannover, Germany. ${ }^{5}$ Dept of Diagnostic and Interventional Radiology, Translational Lung Research Center Heidelberg (TLRC). Heidelberg. Germany. ${ }^{6}$ Member of German Center for Lung Research (DZL), University of Heidelberg, Heidelberg, Germany. ${ }^{7}$ Dept of Cardiothoracic, Transplantation and Vascular Surgery, Hannover Medical School, Hannover, Germany.

${ }^{8}$ Laboratory of Adaptive and Regenerative Biology, Harvard Medical School, Brigham and Women's Hospital, Boston, MA, USA. ${ }^{9}$ Clinic of Pneumology, Hannover Medical School, Hannover, Germany. ${ }^{10}$ These authors contributed equally and share first and the last authorship, respectively.

Correspondence: Maximilian Ackermann, Institute of Functional and Clinical Anatomy, University Medical Center of the Johannes Gutenberg University Mainz, Johann-Joachim-Becher-Weg 13, 55128 Mainz, Germany.

E-mail: maximilian.ackermannduni-mainz.de 
predominant and clinically important patterns/entities of UIP, nonspecific interstitial pneumonia (NSIP) and alveolar fibroelastosis (AFE).

\section{Methods}

Patient selection, specimens and study groups

Fresh lung explants of 24 patients with different subtypes of ILD were included in this study. All diagnoses were made by experienced pulmonary pathologists following the European Respiratory Society and American Thoracic Society guidelines. Patient groups consisted of patients with characteristic radiological and histological patterns: UIP ( $n=6,0$ females, mean \pm SD age at transplantation $61.4 \pm 6.8$ years), idiopathic $\operatorname{NSIP}(\mathrm{n}=6,4$ females, age at transplantation $52.3 \pm 9.2$ years) and AFE ( $n=6,5$ females, age at transplantation $40.7 \pm 27.8$ years) in the context of chronic graft versus host disease, following radio-/ chemotherapy, as the histological hallmark of chronic lung allograft dysfunction and idiopathically (idiopathic pleuroparenchymal fibroelastosis). As controls, we used surgically resected lung allograft samples which had been obtained immediately prior to transplantation for donor-recipient size matching ( $\mathrm{n}=6,1$ female, age at transplantation $29.0 \pm 22.8$ years). Despite careful specimen selection, a significant difference in age between UIP and controls remains ( $p$-value $0.046(<0.05)$, Holm-Bonferroni method). All IPF/UIP patients showed varying degrees of mild pulmonary hypertension consistent with sclerosis of the elastic and muscular pulmonary arteries. Tissue specimens and samples were handled anonymously according to the principles expressed in the Declaration of Helsinki. The study was designed and performed following the requirements of the local ethics committee at Hannover Medical School (ethics vote no. 2702-2015). Sampling and histology, microvascular corrosion casting, micro-computed tomography and gene expression are described in the supplementary methods.

Gene expression analysis of ILD explants

All mRNA expression data was obtained via the nCounter ${ }^{\circledast}$ Analysis System (NanoString Technologies, Seattle, WA, USA) using the PanCancer Progression Panel (770 genes including 30 reference genes). Normalisation of raw counts was performed using the nSolver ${ }^{\mathrm{TM}}$ analysis software version 3.0 (NanoString Technologies) and a modified version of the nCounter ${ }^{\circledast}$ advanced analysis module (version 1.1.5). The normalisation process included positive normalisation (geometric mean), negative normalisation (arithmetic mean) and reference normalisation (geometric mean) using the five most suitable reference genes from the total of 30 available reference genes selected by the geNorm algorithm [24].

\section{Protein expression analysis of ILD explants}

Detection of expressed proteins was done using standard immunohistochemistry and multiplex labelling using the OPAL 7 system. Additional information can be found in the supplementary methods.

\section{Functional analysis}

In order to gain insight on the regulation of physiological functions conveyed by the mRNA expression in different ILD entities, we made use of the Ingenuity Pathway Analysis tool (IPA; Qiagen, Hilden, Germany) [25]. We considered $\mathrm{p}$-values significant according to the following levels of confidence: $\mathrm{p}<0.05$, $\mathrm{p}<0.01$ and $\mathrm{p}<0.001$. Additional information can be found in the supplementary methods.

\section{Statistical analysis of vascular morphometry}

Analysis of vascular morphometry was based on measurements in $\geqslant 24$ lung samples (intervascular distances: 2566 measuring points; vessel diameters: 2259 measuring points). Intervascular distances and vessel diameters were analysed by a one-way ANOVA. If the ANOVA indicated significant differences $(\mathrm{p}<0.05)$ between samples within a group, an all-pairwise analysis was performed (Tukey). Commercially available statistical analysis software (JMP ${ }^{\star}$ 8.0.2.2; SAS Institute Inc., Cary, NC, USA) was used to perform the statistical tests.

\section{Results}

UIP, NSIP and AFE present a specific vascular morphogenetic pattern

In contrast to regular lung morphology (figure 1a-c), histopathological subtypes of ILDs reveal specific morphologic alterations. A characteristic feature of the UIP pattern is a transition between a spatially and temporally heterogeneous interstitial fibrosis, driven by aggregates of myofibroblasts (fibroblast foci), and morphologically normal lung tissue (figure 1f). UIP shows patchy and dense fibrosing remodelling predominantly in the subpleural spaces (figure 1d and e). NSIP shows homogeneous and mild interstitial fibrosis with a uniform and diffuse thickening of alveolar septa as well as some degree of interstitial inflammation (figure 1g-i). The development of AFE involves three stages [26, 27]. The AFE pattern (figure $1 \mathrm{j}-\mathrm{l}$ ) is characterised by preserved elastic fibres outlining the former alveolar septa and a 

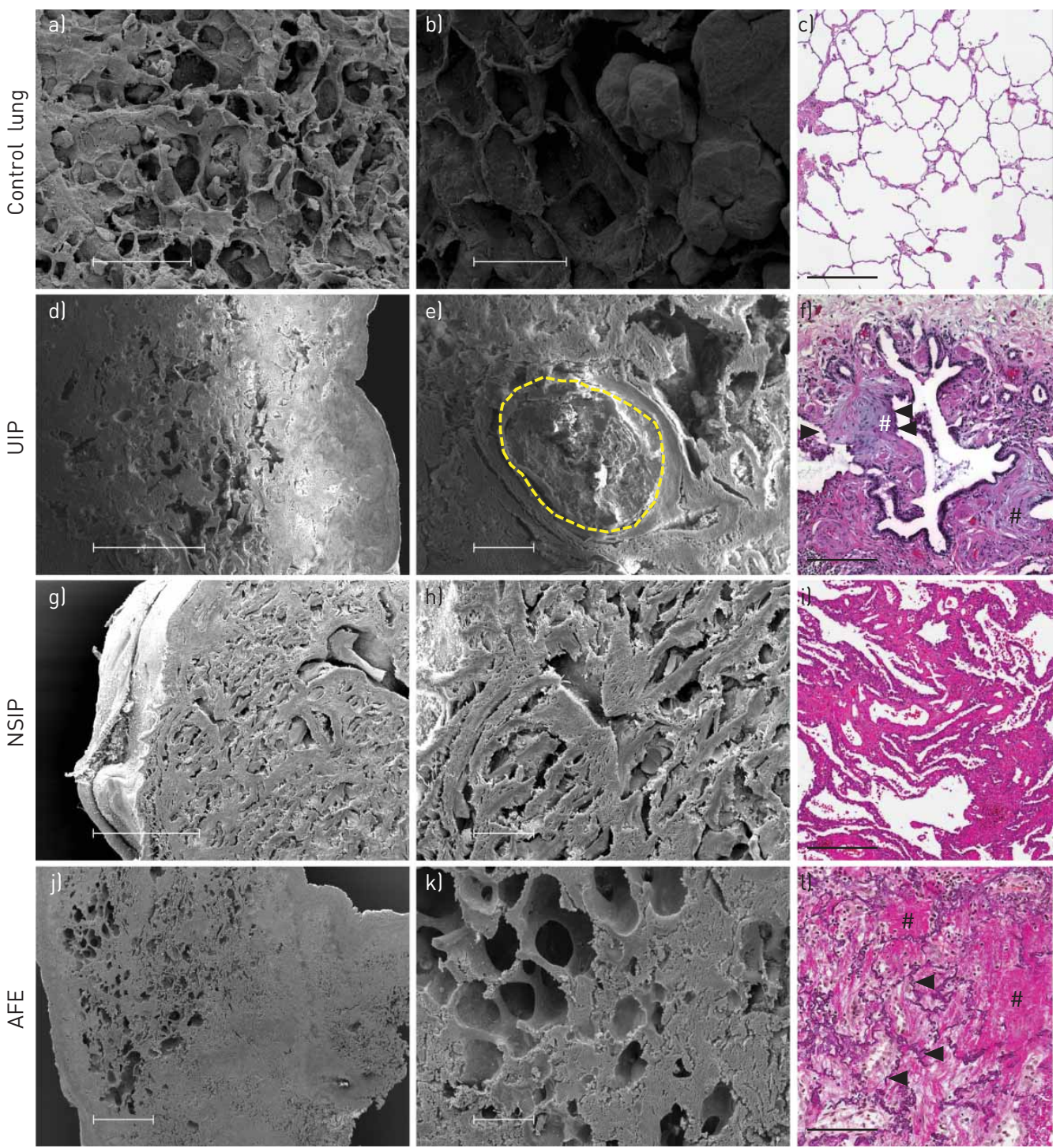

FIGURE 1 Different morphological patterns in usual interstitial pneumonia (UIP), nonspecific interstitial pneumonia (NSIP) and alveolar fibroelastosis (AFE) lungs. Scanning electron micrographs and histological sections demonstrate the injury-specific morphological pattern. a-c) In healthy control lungs, lung architecture includes thin-walled alveolar ducts and alveoli, with sparse bronchial mucous remnants. $d-f)$ The cardinal features of UIP represent a loss of architecture with d) heterogeneous, patchy parenchymal, mostly subpleural fibrotic involvement and fibroblastic foci which are indicative of the progression of the fibrosis; e) adjoining the fibrotic foci, organised thrombotic occlusion (yellow dotted line) is frequently observed in the draining veins; f) adjacent to fibroblastic foci (\#), perifocal alveolar type I cells are replaced by hyperplastic alveolar type II cells (arrowheads). g-i) NSIP is characterised by a diffuse and uniform injury pattern with a moderate fibrotic remodelling of alveolar septa with an interstitial infiltration by lymphocytes, histiocytes and plasma cells (i). j-l) In contrast to conventional fibrotic remodelling, the development of alveolar fibroelastosis (AFE) involves three stages: 1) the "fibrin stage" with fibrin filled alveoli in response to an initial alveolar epithelial damage; 2) the "histiocytic stage" with degradation of alveolar fibrin exudates by local macrophages and accumulation of fibroblasts followed by deposition of collagen within the alveolar space; and 3) the "full developed stage" of AFE presents a concomitant elastosis of alveolar septa (I) (arrowheads), whereas alveolar spaces are consolidated with collagenous fibres (I) (\#). Scanning electron microscopy was performed using a Philips XL30 microscope (Philips, Eindhoven, the Netherlands) at $15 \mathrm{keV}$ and $21 \mu \mathrm{A}$. Hematoxylin and eosin staining ( $c, f$ and i), and Elastica van Gieson staining (I). Scale bars $=200 \mu \mathrm{m}$ ).

continuously increased deposition of elastin in the alveolar interstitium, whereas the airspaces are completely filled up by extracellular matrix.

The immunohistochemical analysis allows a morphological characterisation of fibrotic areas where an expression of the endothelial marker CD31, the bronchoepithelial marker CK7 and the lymphatic marker 
podoplanin can all be frequently observed in close spatial proximity (figure 2). However, immunohistochemical staining of the fibrotic areas cannot reflect the three-dimensional architectural aspects of fibrotic and vascular remodelling.

Our analysis of microvascular corrosion casts of UIP, NSIP and AFE lungs show the underlying morphological patterns (supplementary material 1). The microvascular architecture of UIP lungs reveals a tumour-like and chaotic aberrant distribution of blind-ending, partly occluded sinusoid-like vessels with a lack of hierarchy and wide alterations of vessel diameters with reduced vessel density (figure $3 \mathrm{~b}$ ). The NSIP pattern is characterised by densely packed, dilated tufts of vessel formation with abnormal tortuosity within the alveolar septa (figure 3c). The AFE pattern exhibits a certain resemblance with the NSIP pattern whereby the microvasculature in the preserved alveolar septa suggests some degree of compression (figure 3d).

\section{Vascular morphometry reveals significant alterations in vascular remodelling in UIP, NSIP and AFE}

We assessed the microvascular unit of fibrotic areas by three-dimensional morphometry of the microvascular corrosion casts. The individual vessel diameters in the fibrotic foci, in alveolar vessels of UIP, NSIP, AFE and healthy control lung revealed highly significant differences (figure $4 \mathrm{a}$ ). The highest vessel diameters were seen in UIP lungs (mean \pm SD $16.5 \pm 7.7 \mu \mathrm{m})$ compared with NSIP lungs $(12.7 \pm 5.1 \mu \mathrm{m} ; \mathrm{p}<0.001)$, AFE lungs $(13.1 \pm 5.1 \mu \mathrm{m} ; \mathrm{p}<0.001$, ANOVA) or healthy control lungs (mean $5.9 \pm 1.9 \mu \mathrm{m} ; \mathrm{p}<0.001$, ANOVA). The mean intervascular distances (distance between adjacent individual vessel segments) yielded similar significant results. The highest intervascular distances were observed in UIP lungs $(59.4 \pm 24.1 \mu \mathrm{m})$ in comparison to NSIP lungs $(18.8 \pm 9.0 \mu \mathrm{m} ; \mathrm{p}<0.001$, ANOVA), AFE lungs $(11.6 \pm 4.1 \mu \mathrm{m} ; \mathrm{p}<0.001)$ or the healthy controls $(12.0 \pm 3.7 \mu \mathrm{m} ; \mathrm{p}<0.001)$. Vascular volumetry was conducted on micro-computed tomography of microvascular corrosion casts (supplementary material $2 \mathrm{a}-\mathrm{c}$ ) to assess the vascular changes of upstream vasculature with a diameter $>50 \mu \mathrm{m}$ (figure 4c). UIP lungs showed a higher, albeit significant, vascular volume (36.1 $\pm 5.9 \% ; \mathrm{p}=0.013)$ and vascular surface-volume ratio $(0.43 \pm 0.7 ; \mathrm{p}=0.055$, ANOVA) compared with NSIP lungs (18.9 $\pm 2.0 \%$ and $0.54 \pm 0.6$, respectively), AFE lungs (mean $16.6 \pm 3.7 \%$ and $0.39 \pm 0.04$, respectively) and controls $(6.24 \pm 0.8 \%$ and $0.58 \pm 0.11$, respectively) (data not shown).

\section{Intussusceptive and sprouting angiogenesis accelerate fibrotic neovascularisation}

We identified intussusceptive and sprouting angiogenesis within the microvascular corrosion casts of all analysed lungs. The formation of intussusceptive pillars was progressed by initial vasodilation followed by incorporation of CD34/31-positive progenitor-like $[28,29]$ as well as the activation of mesenchymal stem cells which span a transluminal bridge, the "intussusceptive pillar" (figure 5a and b) (supplementary material 5; quantification of proliferation of CD34/CD31 positive cells by Ki-67). The occurrence of

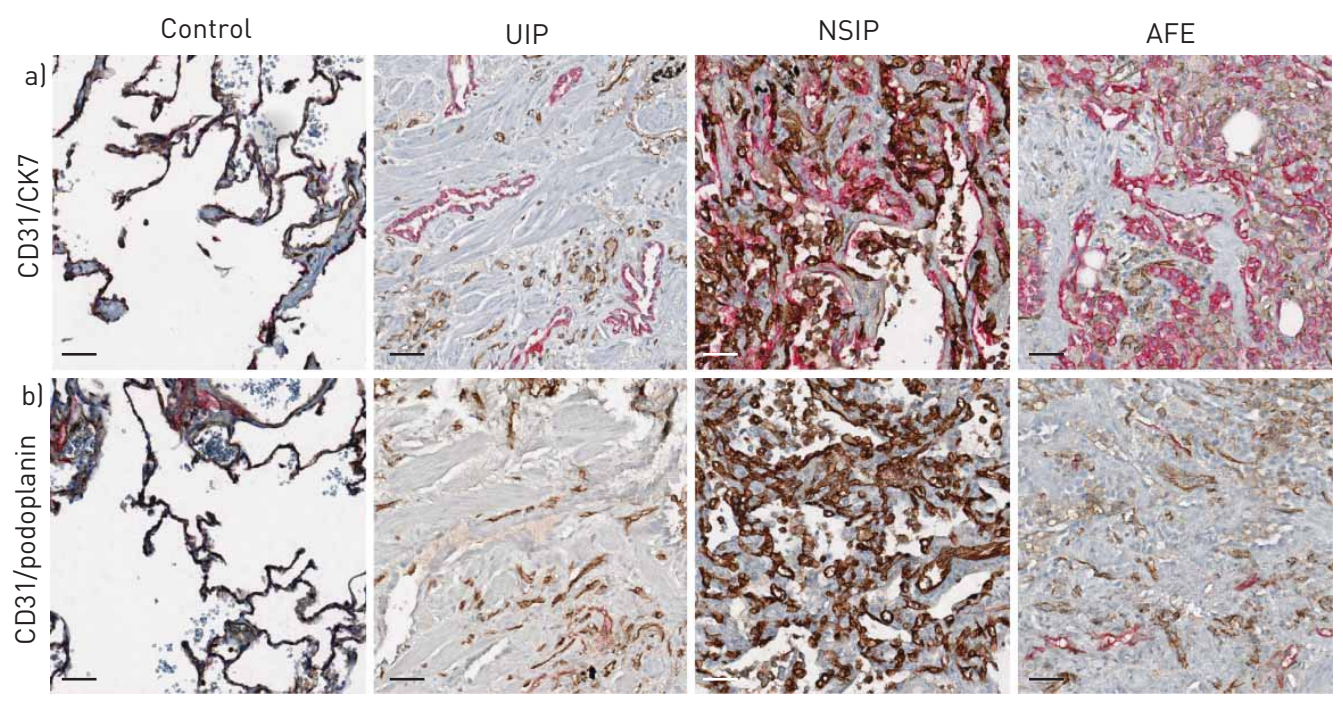

FIGURE 2 Immunohistochemical detection of CD31, CK7 and podoplanin expression in alveolar fibroelastosis (AFE), nonspecific interstitial pneumonia (NSIP) and usual interstitial pneumonia (UIP). Double-staining against the endothelial marker CD31 (brown) and a) the bronchoepithelial marker cytokeratin CK7 (red) and b) the lymphatic marker podoplanin (red). Staining results demonstrate the spatial heterogeneity of vascularisation in the fibrotic areas in AFE, NSIP and UIP, as compared to controls. Scale bars $=50 \mu \mathrm{m}$. 

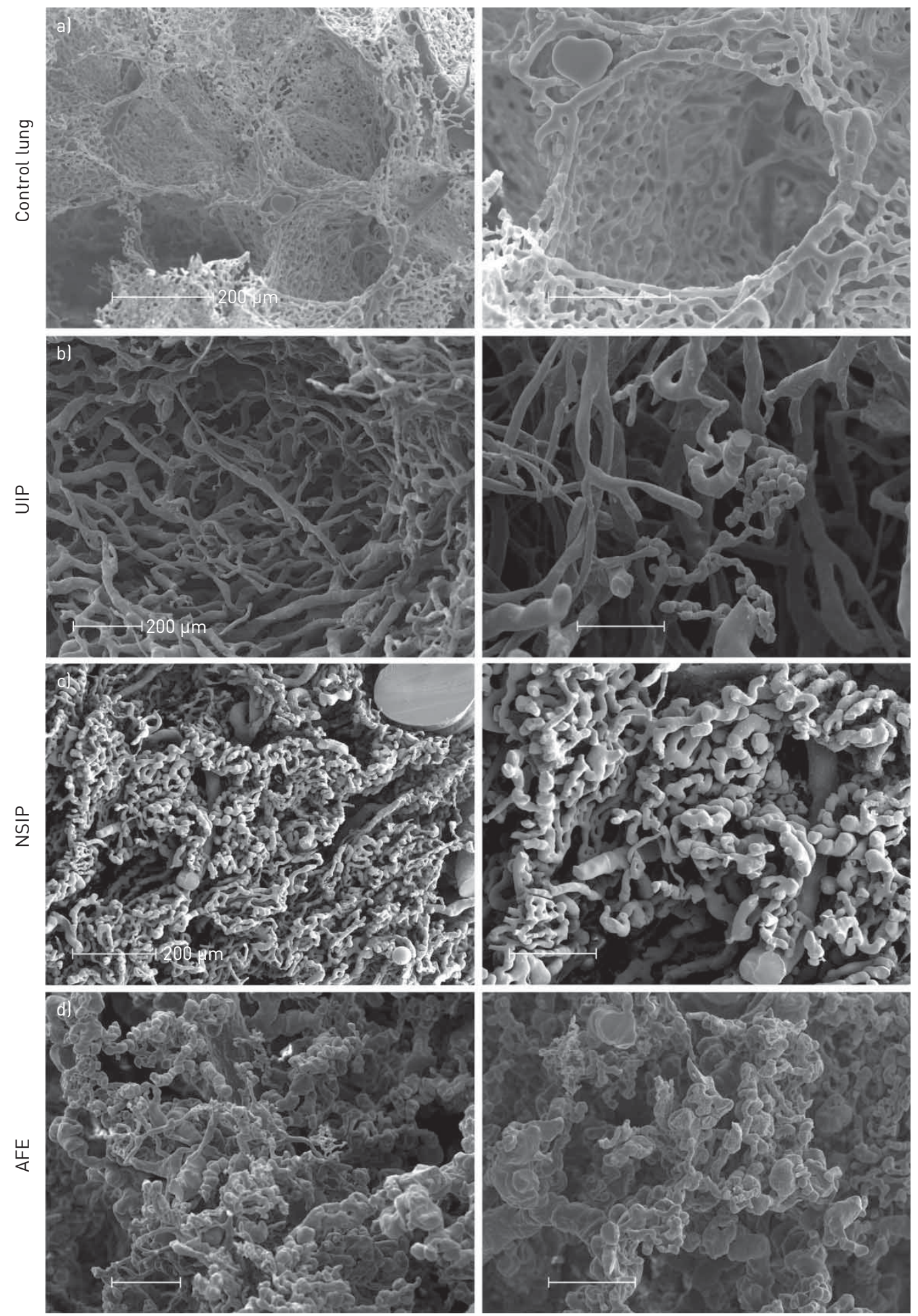

FIGURE 3 Microvascular architectural alterations in usual interstitial pneumonia (UIP), nonspecific interstitial pneumonia (NSIP) and alveolar fibroelastosis (AFE). Scanning electron micrographs of microvascular corrosion casts illustrate the substantial architectural differences between the different injury patterns. a) Healthy control lung vasculature is characterised by thin-walled alveolar capillary plexuses aligned along the alveolar duct; b) UIP lungs demonstrate an aberrant vasculature with blunt, sinusoid-like vessels, without a clear vessel hierarchy, but instead high variability of vessel diameters and small vessel sprouts; c) NSIP lungs present with dense, tortuous dilated tufts of vessel formations in the alveolar septa and frequent intussusceptive pillars; d) AFE lungs resemble the appearance of the NSIP lungs with pronounced vascular alterations in the alveolar septa. Scale bars $=100 \mu \mathrm{m}$, unless otherwise stated. 
a)

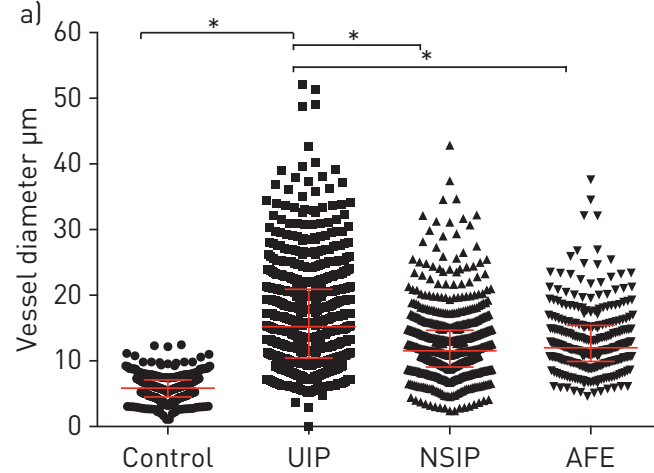

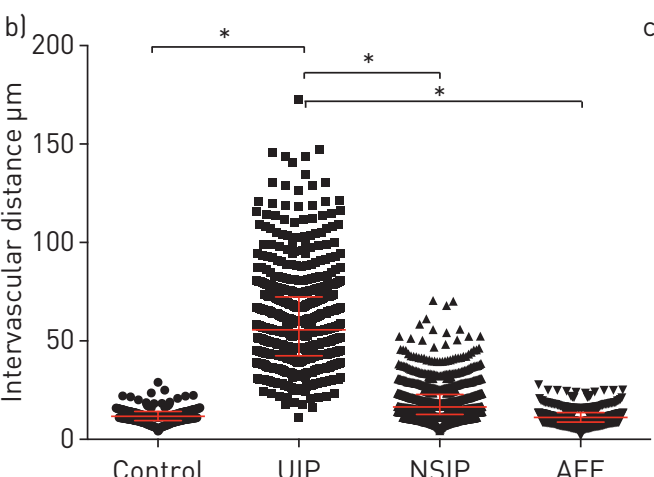

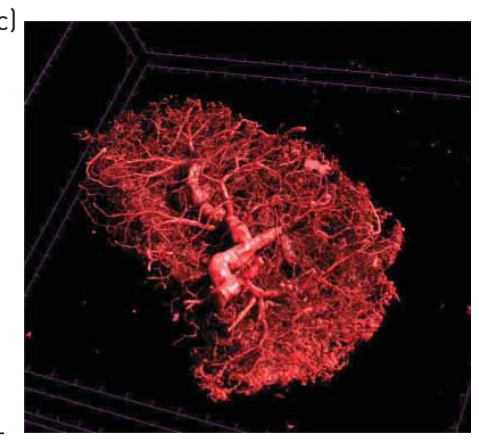

FIGURE 4 Vascular morphometry of usual interstitial pneumonia (UIP), nonspecific interstitial pneumonia (NSIP) and alveolar fibroelastosis (AFE). The morphometrical assessment was carried out in the alveolar plexus of fibrotic areas in UIP, NSIP and AFE, and in healthy control lungs. a) Vessel diameters and b) intervascular distances showed significant differences between the different injury patterns. Scatter plots show the respective median and the 25 th and 75 th percentile in red. ${ }^{* * *}: p<0.001$ (ANOVA). c) Micro-computed tomography on corrosion casts were performed to assess the volumetric changes of upstream vasculature. Only vascular changes of $>50 \mu \mathrm{m}$ diameter were evaluated. Three-dimensional visualisation of microvascular corrosion casts shows exemplarily the upstream vascular hierarchy of a NSIP lung.

sprouting and intussusceptive angiogenesis was observed in all fibrotic lungs, whereas NSIP lungs showed significantly higher frequencies of pillar formations in comparison to UIP and AFE lungs (figure 5c; $\mathrm{p}<0.001$, ANOVA). In contrast to intussusception, the highest frequencies ( $>10$-fold increase) of sprout formation were seen in UIP lungs compared to NSIP and AFE lungs ( $p<0.001$, ANOVA) (figure $5 c$ ). The high occurrence of intussusceptive angiogenesis in NSIP lungs was predominantly observed in the

a)

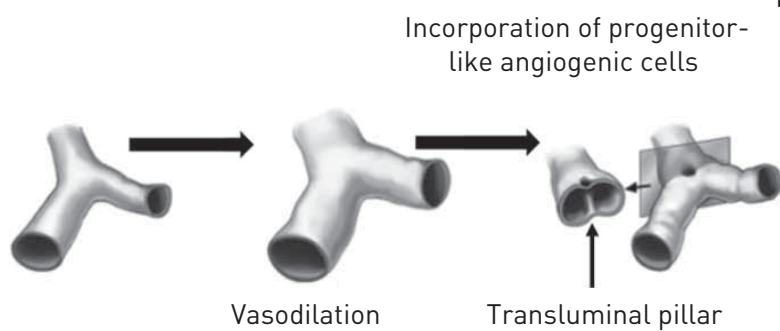
flow changes
C)

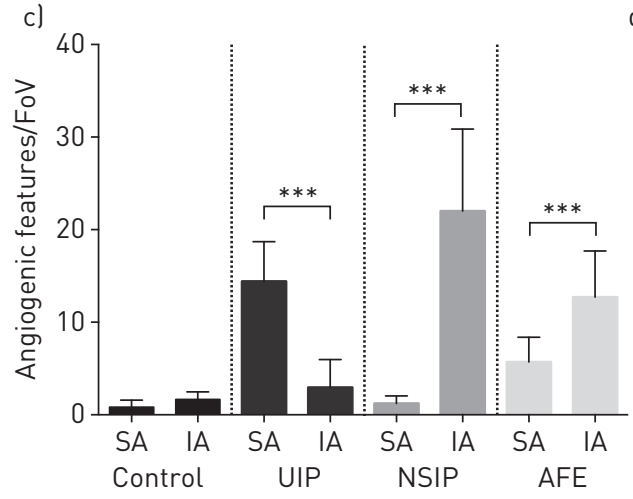

b) Intussusceptive angiogenesis

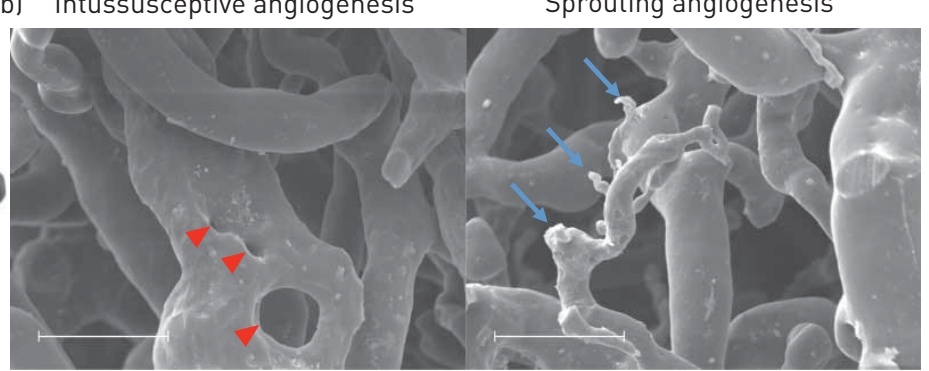

d)

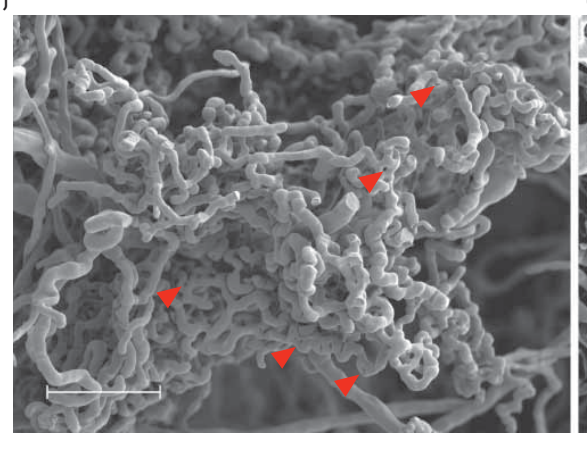

e)

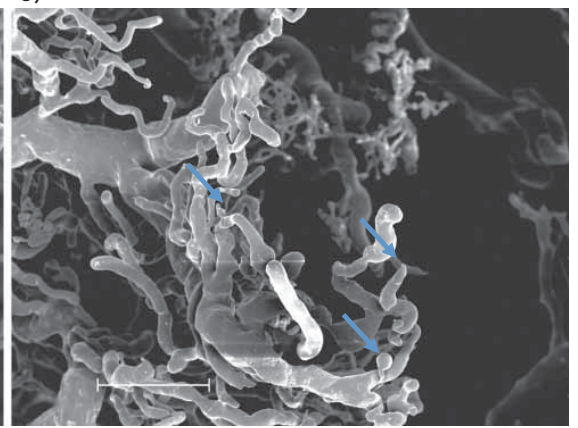

FIGURE 5 Neoangiogenic remodelling of fibrotic lung tissue by intussusceptive and sprouting angiogenesis. a) Schematic representation of intussusceptive angiogenesis. Blood flow and velocity are reduced by marked vasodilation. After the dilation of the vessel, intraluminal pillars form at vessel bifurcations by an intraluminal intussusception of myofibroblasts and incorporation of tissue resident or blood-borne progenitor-like angiogenic cells. b) The intussusceptive transluminal pillar, a tissue bridge that leads to multiple septations of the vessel lumina, appears as a "hole" in microvascular corrosion cast (arrowheads) whereas sprouting angiogenesis forms little extraluminal "sprouts" (arrows) from pre-existing blood vessels; scale bars $=100 \mu \mathrm{m}$. c) The numerical density newly formed sprouts (SA) and intussusceptive pillars (IA) per field of view (FoV) assessed on microvascular corrosion casts revealed the highest frequency of intussusceptive pillars in nonspecific interstitial pneumonia (NSIP) lungs, whereby the highest occurrence of sprouts was observed in usual interstitial pneumonia (UIP) lungs. $* * *$ : p<0.001 (ANOVA). d) The septal thickening in NSIP lungs is mainly characterised by a dense vascular network which expands by numerous intussusceptive

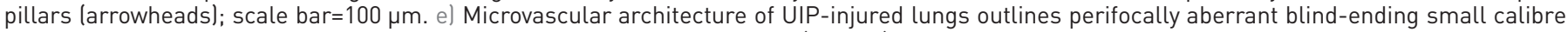
vessels with vessel sprouts which are aligned towards the fibroblastic foci (arrows); scale bar: $200 \mu \mathrm{m}$.AFE: alveolar fibroelastosis. 
expanded septa, in which a prominent expansion of tuft-like blood vessels was observed (figure $5 \mathrm{~d}$ ). In the UIP lungs, blunt, blind-ending vessels with dilated parent venules were located adjacent to fibroblastic foci. At the tip of these blunt vessels, sprouts were mostly aligning centrally towards the fibroblastic foci (figure $5 e)$.

\section{Differential regulation of mRNA expression and biological functions in UIP, NSIP and AFE}

We performed a digital multiplexed gene expression analysis via NanoString nCounter technology (supplementary material 3a-d). Transcript analysis showed significant differences in the expression of 20 genes among the three lung injury patterns compared to healthy control lungs (figure 6a). For the sake of validation, the protein expression of selected genes was also analysed in a compartment specific manner via immunohistochemistry. Here, collagens (COL I, III, IV) and collagen remodelling matrix metalloproteinases (MMP2, MMP9, MMP14) correlated well with the mRNA expression in the corresponding sites of neoangiogenesis (supplementary material 4). Comparative gene analysis demonstrated an overlap of statistically significant differentially expressed genes between UIP, NSIP and AFE (figure 6b). 16 genes were significantly upregulated in AFE lungs, whereas only four genes were differentially expressed in UIP lungs and one gene in NSIP lungs. One gene (IGF1) was significantly upregulated $(\mathrm{p}<0.001)$ as a common denominator of AFE and UIP, whereas MEG3 was significantly $(\mathrm{p}<0.01)$ expressed in both injury patterns of UIP and NSIP. COL1A1 and THY1 are highly significantly $(p<0.001)$ expressed in NSIP and AFE lungs (supplementary material 3). In addition to canonical pathways, differentially expressed genes were also categorised to related functional pathways using IPA (figure 6c). By this approach, AFE lungs exhibited an upregulation of different function pathways compared to NSIP and UIP. The analysis argued for significant $(\mathrm{p}<0.01)$ and highly significant $(\mathrm{p}<0.001)$ activation of epithelial cell and fibroblast migration, as well as macrophage differentiation in AFE and NSIP lungs. Moreover, activation of epithelial-mesenchymal transition was observed in AFE lungs. Neoangiogenesis, migration of endothelial cells and fibrogenesis were also significantly activated in AFE compared to UIP lungs $(\mathrm{p}<0.01)$. In contrast to AFE, the lungs of NSIP and UIP showed that the pathways are less activated (NSIP $>$ UIP). In the analysis, signalling pathways of inflammation are only upregulated in UIP lungs in comparison to AFE and NSIP.

\section{Discussion}

In this study, we assessed the morphogenetic and molecular aspects of angiogenesis in the interstitial lung injury patterns of UIP, NSIP and AFE. For this we assembled patient cohorts which showed no significant differences with regard to age or sex, with the exception of the UIP group (older, in general). However, since the statistical analysis of the gene expression data of the healthy control group revealed no age-dependent effects, it seems plausible that NSIP, AFE and UIP could be analysed in comparison to the healthy control group.

First, our data demonstrate that the histopathological morphological features widely depend on the underlying vascular architectural alterations. NSIP and AFE reveal a similar vascular injury pattern with pronounced remodelling in the thickened alveolar septa, whereas UIP lungs are characterised by upregulation of vascular density in the upstream vessels and a reduction of vessel density in the perifocal and alveolar perifocal capillary plexus. Furthermore, the difference in vessel diameters and intervascular distances reflect these individual changes. Second, our data provide evidence for a role of sprouting and intussusceptive angiogenesis in the expansion of perifocal and alveolar blood vessels. Third, our findings on differences and similarities of angiogenesis-related gene expression underline the morphological features of vascular remodelling in UIP, NSIP and AFE (figure 7).

The impact of angiogenesis on the pathogenesis and progression of ILD has become a subject of controversy $[12,20,25]$. While several studies proposed that stimulated aberrant angiogenesis is a core characteristic of ILD, other authors have reported on reduced vascular densities in lung biopsies of IPF patients, in particular $[5,20,30]$. The legitimate question "angiogenesis in pulmonary fibrosis - too much or not enough?" raised by HANUMEGOWDa et al. [12] cannot be easily addressed due to the architectural complexity of the vascular system and its density on two-dimensional histological slides. Therefore, in this study, using three-dimensional analysis, we put effort into unravelling the complexity of both spatial and temporal heterogeneity of microvascular changes present in UIP, NSIP and AFE lungs. It seems contradictory that the overall vascular volume in UIP is upregulated, whereas the perifocal vascular density is reduced and thinned out while the exact opposite was evident with NSIP lungs. The predictive clinical value of vascular volume as a marker for mortality was recently discussed in depth by JАCOB et al. [19], using a computer-based algorithm to assess pulmonary vascular volumes measured by computed tomography. However, this technique only captures the alterations of upstream blood vessels from the alveolar and perifocal capillary plexus, but does not address the heterogeneous vascular morphological aspects of newly formed blood vessels that probably contribute to the progression of the disease. The 

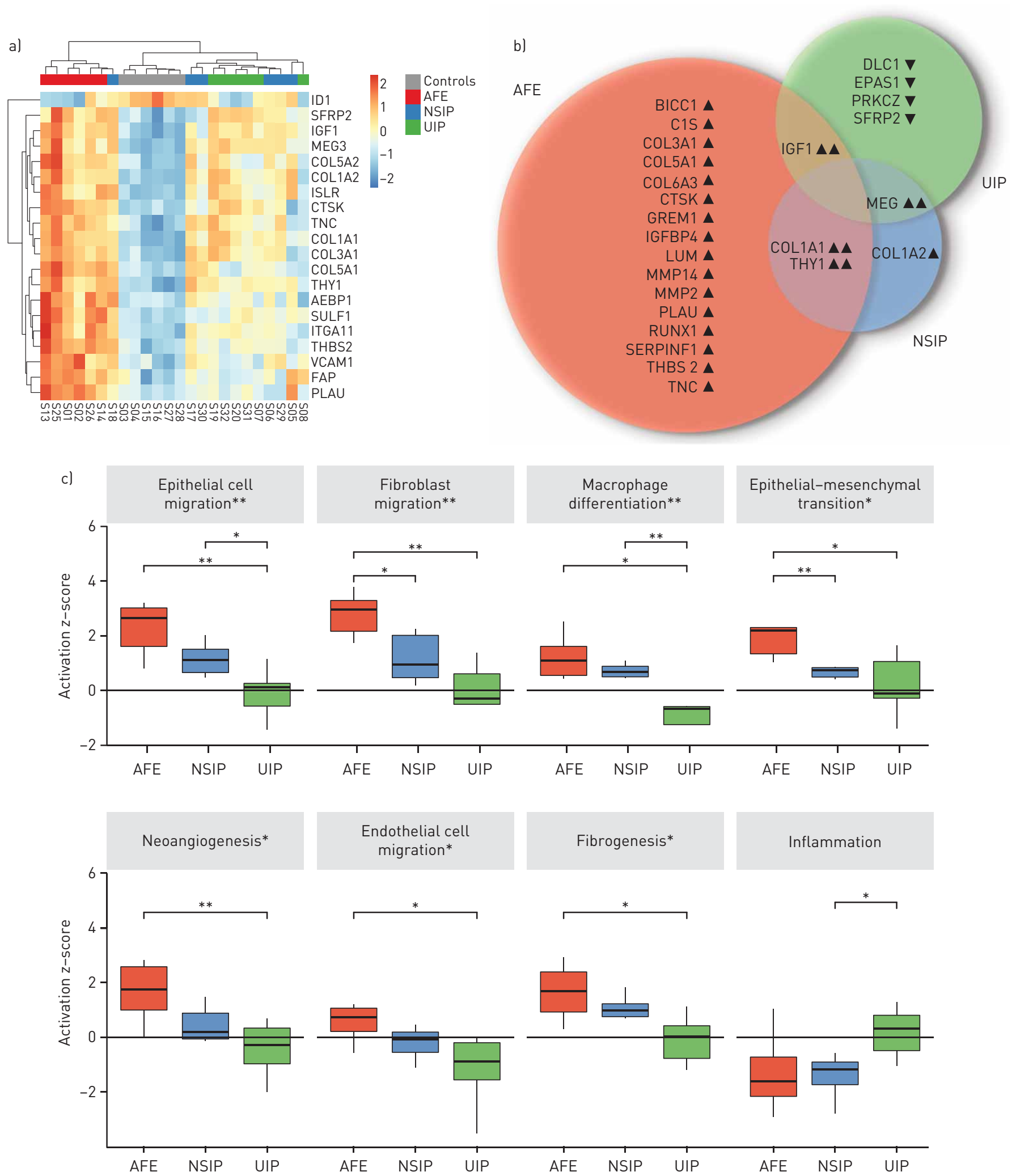

\section{AFE}

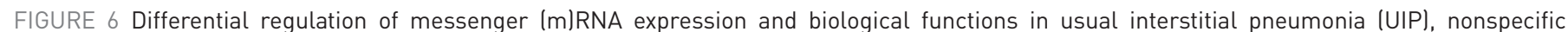

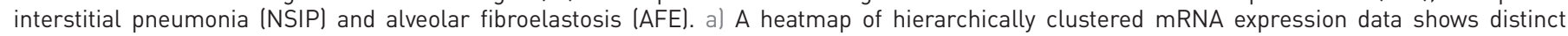

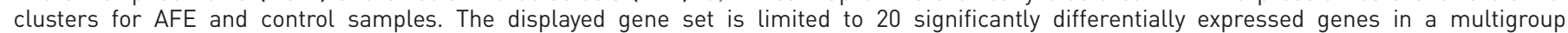

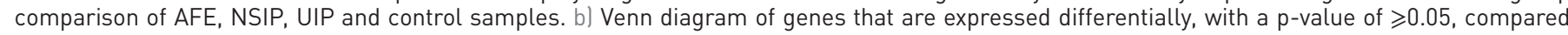

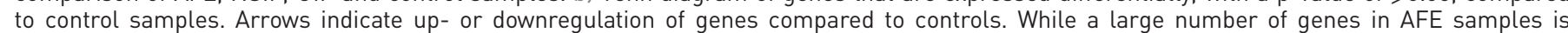

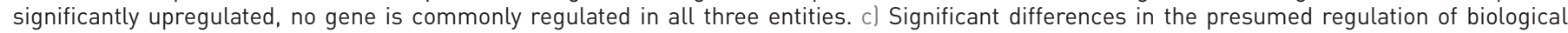
functions. Significance level in a multigroup test of all three entities: ${ }^{*}: p<0.05 ;{ }^{* *}$ : $p<0.01$. AFE and UIP frequently exhibit significant differences. 

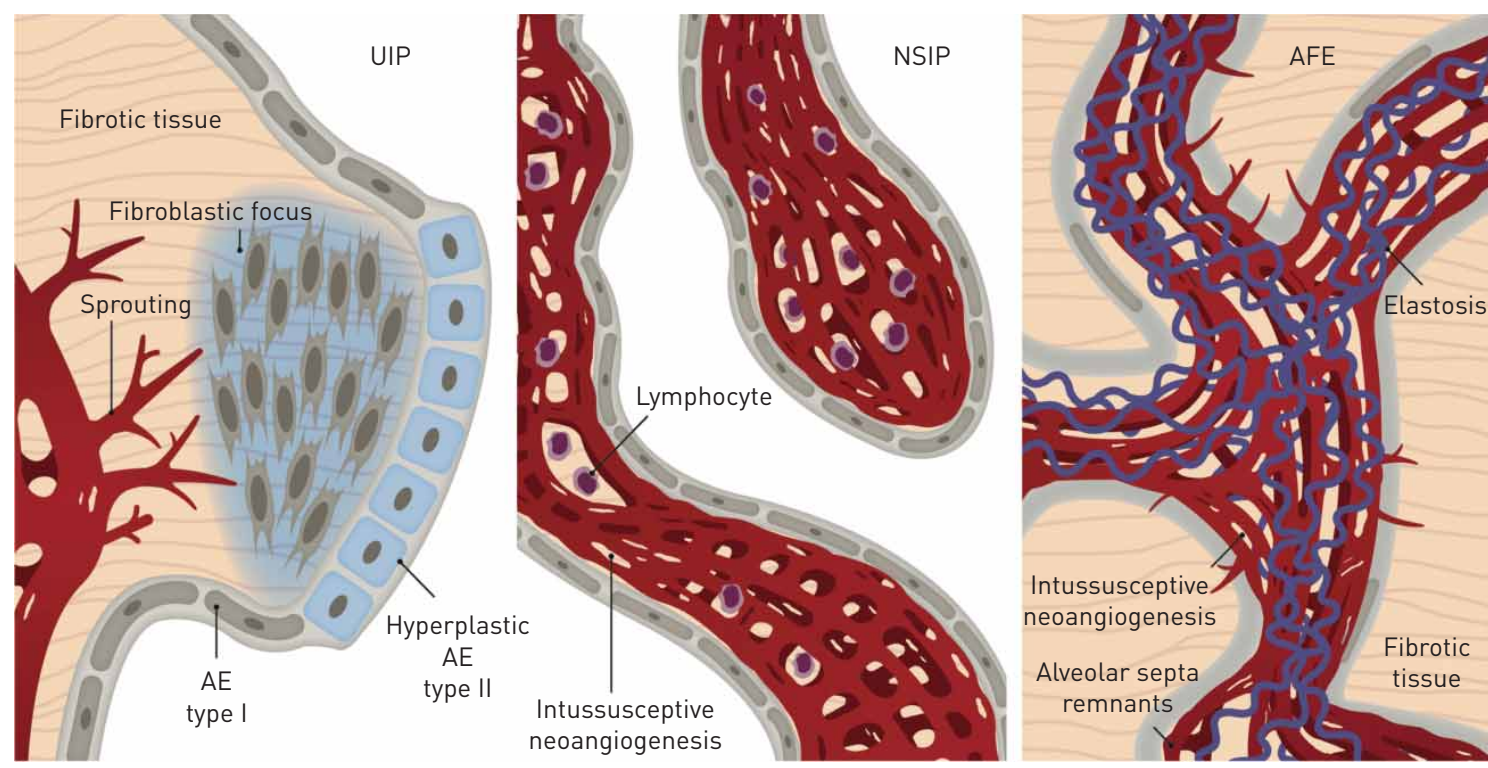

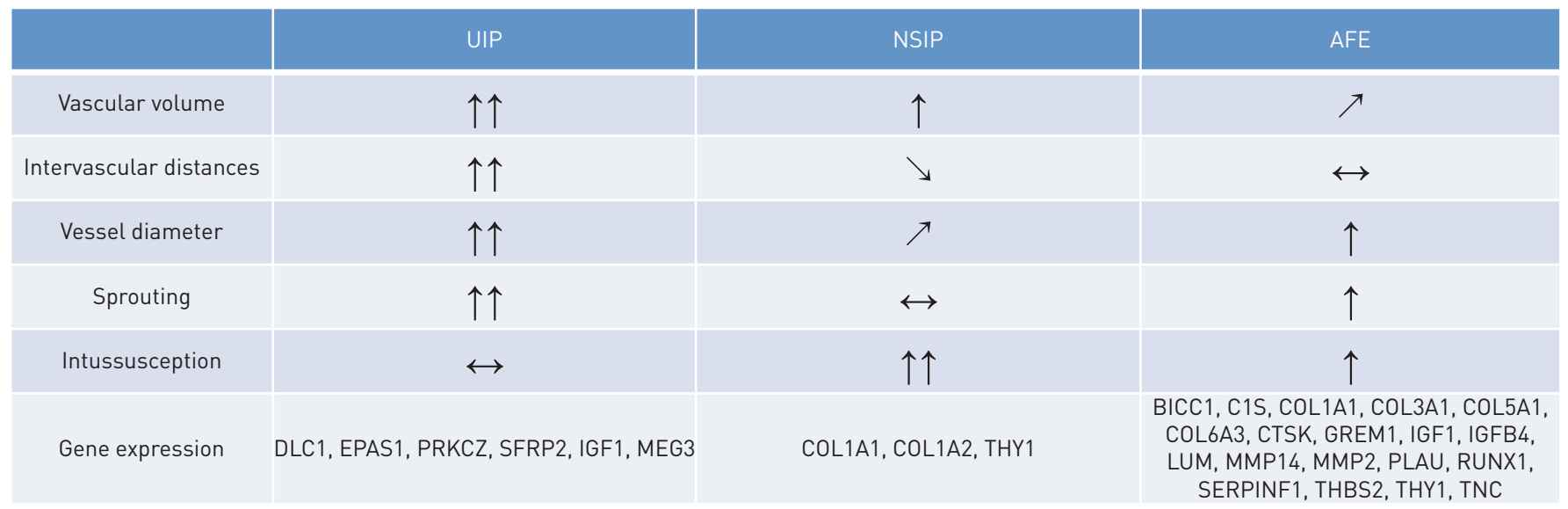

FIGURE 7 Schematic illustration of morphomolecular motifs in histopathological subtypes usual interstitial pneumonia (UIP), nonspecific interstitial pneumonia (NSIP) and alveolar fibroelastosis (AFE). UIP is characterised by prominent basophilic fibroblastic foci with adjacent hyperplastic type 2 alveolar epithelial (AE) cells. Here, we frequently find newly formed blood vessels via sprouting reaching out towards the fibroblastic foci. In NSIP thickened septa are composed of blood vessels with intussusceptive pillars and increased amounts of lymphocytes. In AFE former alveolar air spaces are filled with collagen, while former alveolar walls are remodelled, showing elastosis and increased amounts of intussusceptive blood vessels.

formation of fibrotic areas leads inevitably to haemodynamic changes with an opening of anastomoses between the pulmonary and bronchial circulation. These haemodynamic and micromechanical forces are known to enhance the expansion of vascular plexuses by intussusceptive angiogenesis [26, 31]. Thus, this pillar formation and branch remodelling may represent a pivotal adaptive response to the continually increasing blood flow and blood pressure during inflammation and regeneration. Previously, it has been shown by our group that chronic inflammation triggers the migration and recruitment of progenitor-like angiogenic cells to the site of remodelling [25-27]. In the inflammatory ILD patterns of NSIP and AFE $[32,33]$, we observed a frequent occurrence of intussusceptive pillars.

In contrast, angiogenesis in UIP lungs was driven by hypoxia with perifocal sprouting angiogenesis, which, among other factors, was indicated by the upregulation of insulin-like growth factor (IGF)-1 [34]. Comparable to malignant tumours, the UIP-specific vascularity showed a chaotic vessel-like arrangement with numerous blunt ends. This hypoxia-induced vascular remodelling is associated with the modification of the extracellular matrix by MMPs, and as we showed earlier, inhibition of IGF-1 can impede sprouting angiogenesis significantly [35]. In addition, localised hypoxia results in metaplasia and hyperplasia of alveolar type II cells by Notch signalling and $\mathrm{Krt} 5^{\text {pos }}$ basal-like cell expansion in alveolar repair and remodelling [36].

Nintedanib, a multikinase inhibitor with anti-angiogenic properties, has been shown to have efficacy in reducing the decline of lung function in IPF patients [37]. Besides anti-inflammatory and anti-fibrotic effects, 
the question remains whether an anti-angiogenic therapy may play a pivotal role in improving the functional outcome in IPF patients. Our own studies in a bleomycin-induced mouse fibrosis model demonstrated that the altered vascular architecture appears to be "normalised" after nintedanib treatment [16], a phenomenon also found in tumours treated with an anti-angiogenic therapy [35, 38]. Moreover, intervascular distances and vessel diameters showed significant differences in nintedanib-treated animals, whereas lung function improved under the therapy. Therefore, the structure-function dynamics of microvascular architecture are likely to underlie the injury and progression patterns of ILD. Otherwise, FARKAS et al. [39] could show in an IPF rat model with transient overexpression of active TGF- $\beta 1$ that a decreased vascularity is associated with an extensive remodelling and a secondary pulmonary hypertension. In this model, the administration of VEGF showed an amelioration of pulmonary hypertension via inhibition of endothelial apoptosis, yet also an aggravation of pulmonary fibrosis.

Some authors suggest that vascular alterations might represent an initial trigger in the pathogenesis and the progression of ILD [20,40] and conclude that patients with pulmonary fibrosis show aspects of pulmonary venous occlusive disease (PVOD) with aberrant postcapillary expansion by haemangiomatosis. Similar observations were made in non-fibrotic areas of patients with IPF [40], in which $65 \%$ of cases demonstrated occlusive venopathy. Our own recent work [41] on PVOD highlights multifocal intussusceptive neoangiogenesis and vascular sprouting as representing the three-dimensional correlate of progressive pulmonary haemangiomatosis. In addition, we described comparable morphogenetic phenomena in chronic thromboembolic pulmonary hypertension in the form of plexiform vasculopathy with pronounced intussusceptive angiogenesis [42].

NSIP and AFE revealed a shared upregulation of COL1A1 and Thy1/CD90. Thy1/CD90 is expressed on endothelial cells at sites of chronic inflammation and adjacent to malignant neoplasms, as well as on adventitial mesenchymal progenitor cells in adult human arteries, where both are involved in the microvascular expansion by intussusceptive neoangiogenesis [43]. Other studies reported that the intussusceptive capillary growth is the main driver in post-injury angiogenesis in a Thyl.1-nephritis model [43], which is consistent with the recovery of septal blood vessels in human NSIP and AFE lungs. We observed a higher frequency of intussusceptive features in NSIP and AFE fibrotic lungs. The expansion of the microvascular network by intussusception is associated with the recruitment of progenitor-like angiogenic cells forming transluminal pillars $[26,31]$. These circulating progenitor-like angiogenic cells are recruited as tissue-resident or bone-marrow derived monocytes which can transdifferentiate among other types into proangiogenic M2-polarised alveolar macrophages [29, 44, 45] or into endothelial cells which then incorporate into newly formed intussusceptive blood vessels [42, 45]. Alvarez et al. [44] characterisedendothelial cells isolated from the lung microcirculation as highly vasculogenic and capable of renewing the entire hierarchy of endothelial cell growth.

Recent evidence [46] confirmed that bone marrow derived mononuclear cells are major constituents in the vascular expansion by intussusceptive angiogenesis, controlled by SDF-1/CXCR4 signalling. Our group observed similar effects on the migration of $\mathrm{CD}_{11} \mathrm{~b}^{+}$monocytes during lung regeneration [29]. In summary, we provide the first evidence that the histopathological injury patterns of life-threatening ILDs are highly dependent on the underlying vascular architectural alterations and vessel densities in UIP, NSIP and AFE lungs. Whereas UIP lungs showed a higher density of upstream vascularity and lower density in perifocal blood vessels, NSIP and AFE lungs revealed densely packed alveolar and septal blood vessels. Microvascular alterations by sprouting (UIP) and intussusceptive angiogenesis (NSIP, AFE) may contribute as one of the pivotal pathogenetic mechanisms in the progression of disease in the capillaryalveolar interaction. Our data can serve to identify novel therapeutic targets with the potential to favourably alter the course of ILD by influencing aberrant angiogenesis in the different subtypes of ILDs.

Acknowledgements: The authors thank Regina Engelhardt, Annette Mueller Brechlin, Christina Petzold, Nicole Kroenke and Constantin Kloepper (Hannover Medical School, Hannover, Germany) and Kerstin Bahr (University Medical Center Mainz, Mainz, Germany) for their excellent technical support, and Gareth Griffiths, Allison Seidel and Pauline Tittmann (Hannover Medical School) for editing the manuscript.

Author contributions: A. Haverich, T. Welte and D. Jonigk managed the patients. M. Ackermann, A. Haverich, L. Neubert, S. Schubert, H. Stark, W. Stiller, W.L. Wagner and M. Kuehnel worked up surgical specimens. M. Ackermann, H. Stark, S.J. Mentzer, T. Welte, A. Höfer, H.R. Shah, M. Kuehnel and D. Jonigk interpreted the data. M. Ackermann, S. Schubert, H. Stark, M. Kuehnel and D. Jonigk wrote the manuscript. M. Ackermann, L. Neubert, P. Borchert, M. Wielpütz, W. Stiller, W.L. Wagner, F. Linz and H. Stark prepared the images. M. Ackermann, T. Welte, S.J. Mentzer, H. Stark, M. Kuehnel and D. Jonigk revised the manuscript.

Support statement: This work was supported by NIH grants HL94567 and HL134229 to M. Ackermann and S.J. Mentzer; Sonderforschungsbereich, "SFB" 738 (Projekt B9) of the German Research Foundation to D. Jonigk; and the European Research Council (ERC), European Consolidator Grant, XHale to Da. Jonigk (reference number 771883). 
Conflict of interest: H. Stark has nothing to disclose. L. Neubert has nothing to disclose. S. Schubert has nothing to disclose. P. Borchert has nothing to disclose. F. Linz has nothing to disclose. W.L. Wagner has nothing to disclose. W. Stiller reports grants from German Federal Ministry of Education and Research (BMBF), during the conduct of the study. M. Wielpütz reports grants from Boehringer Ingelheim and Vertex Pharma, outside the submitted work. A. Höfer has nothing to disclose. A. Haverich has nothing to disclose. S.J. Mentzer has nothing to disclose. H.R. Shah has nothing to disclose. T. Welte reports grants from German Ministry of Research and Education, during the conduct of the study; personal fees for advisory board work and lectures from Boehringer Ingelheim, personal fees for clinical studies from Roche, outside the submitted work. M. Kuehnel has nothing to disclose. D. Jonigk has nothing to disclose. M. Ackermann has nothing to disclose.

\section{References}

1 Selman M, Pardo A. The epithelial/fibroblastic pathway in the pathogenesis of idiopathic pulmonary fibrosis. Am J Respir Cell Mol Biol 2003; 29: 3 Suppl, S93-S97.

2 Tonnesen MG, Feng X, Clark RA. Angiogenesis in wound healing. J Investig Dermatol Symp Proc 2000; 5: 40-46.

3 Crapo JD, Barry BE, Gehr P, et al. Cell number and cell characteristics of the normal human lung. Am Rev Respir Dis 1982; 126: 332-337.

4 Gehr P, Bachofen M, Weibel ER. The normal human lung: ultrastructure and morphometric estimation of diffusion capacity. Respir Physiol 1978; 32: 121-140.

5 Ebina $M$, Shimizukawa $M$, Shibata N, et al. Heterogeneous increase in CD34-positive alveolar capillaries in idiopathic pulmonary fibrosis. Am J Respir Crit Care Med 2004; 169: 1203-1208.

6 Konerding MA, Gibney BC, Houdek JP, et al. Spatial dependence of alveolar angiogenesis in post-pneumonectomy lung growth. Angiogenesis 2012; 15: 23-32.

7 Tammela T, Zarkada G, Wallgard E, et al. Blocking VEGFR-3 suppresses angiogenic sprouting and vascular network formation. Nature 2008; 454: 656-660.

8 Hlushchuk R, Riesterer $\mathrm{O}$, Baum $\mathrm{O}$, et al. Tumor recovery by angiogenic switch from sprouting to intussusceptive angiogenesis after treatment with PTK787/ZK222584 or ionizing radiation. Am J Pathol 2008; 173: 1173-1185. Epub 2008 Sep 11.

9 Ackermann M, Konerding MA. Vascular casting for the study of vascular morphogenesis. Methods Mol Biol 2015; 1214: 49-66.

10 Tzouvelekis A, Anevlavis S, Bouros D. Angiogenesis in interstitial lung diseases: a pathogenetic hallmark or a bystander? Respir Res 2006; 7: 82.

11 Parra ER, David YR, da Costa LR, et al. Heterogeneous remodeling of lung vessels in idiopathic pulmonary fibrosis. Lung 2005; 183: 291-300.

12 Hanumegowda C, Farkas L, Kolb M. Angiogenesis in pulmonary fibrosis: too much or not enough? Chest 2012; 142: 200-207.

13 Burdick MD, Murray LA, Keane MP, et al. CXCL11 attenuates bleomycin-induced pulmonary fibrosis via inhibition of vascular remodeling. Am J Respir Crit Care Med 2005; 171: 261-268.

14 Richeldi L, du Bois RM, Raghu G, et al. Efficacy and safety of nintedanib in idiopathic pulmonary fibrosis. $N$ Engl J Med 2014; 370: 2071-2082.

15 Rivera-Ortega P, Hayton C, Blaikley J, et al. Nintedanib in the management of idiopathic pulmonary fibrosis: clinical trial evidence and real-world experience. Ther Adv Respir Dis 2018; 12: 1753466618800618,

16 Ackermann M, Kim YO, Wagner WL, et al. Effects of nintedanib on the microvascular architecture in a lung fibrosis model. Angiogenesis 2017; 20: 359-372.

17 Ask K, Tandon K, Ayaub E, et al. Nintedanib prevents IL-6-mediated secretion of CCL-18 in alternatively activated macrophages and transition of fibroblasts to myofibroblasts. Eur Respir J 2017; 50: PA3478. .

18 Tandon K, Herrmann F, Ayaub E, et al. Nintedanib attenuates the polarization of profibrotic macrophages through the inhibition of tyrosine phosphorylation on CSF1 receptor. Am J Respir Crit Care Med 2017; 195: A2397.

19 Jacob J, Bartholmai BJ, Rajagopalan S, et al. Mortality prediction in idiopathic pulmonary fibrosis: evaluation of computer-based CT analysis with conventional severity measures. Eur Respir J 2017; 49: 1601011.

20 Puxeddu E, Cavalli F, Pezzuto G, et al. Impact of pulmonary vascular volume on mortality in IPF: is it time to reconsider the role of vasculature in disease pathogenesis and progression? Eur Respir J 2017; 49: 1602345.

21 Hashimoto N, Phan SH, Imaizumi K, et al. Endothelial-mesenchymal transition in bleomycin-induced pulmonary fibrosis. Am J Respir Cell Mol Biol 2010; 43: 161-172.

22 Jonigk D, Stark H, Braubach $\mathrm{P}$, et al. Morphological and molecular motifs of fibrosing pulmonary injury patterns. J Pathol Clin Res 2019; 5: 256-271.

23 du Bois R, King TE Jr. Challenges in pulmonary fibrosis · 5: the NSIP/UIP debate. Thorax 2007; 62: 1008-1012.

24 Vandesompele J, De Preter K, Pattyn F, et al. Accurate normalization of real-time quantitative RT-PCR data by geometric averaging of multiple internal control genes. Genome Biol 2002; 3: RESEARCH0034.

25 Farkas L, Gauldie J, Voelkel NF, et al. Pulmonary hypertension and idiopathic pulmonary fibrosis: a tale of angiogenesis, apoptosis, and growth factors. Am J Respir Cell Mol Biol 2011; 45: 1-15.

26 Mentzer SJ, Konerding MA. Intussusceptive angiogenesis: expansion and remodeling of microvascular networks. Angiogenesis 2014; 17: 499-509.

27 Konerding MA, Turhan A, Ravnic DJ, et al. Inflammation-induced intussusceptive angiogenesis in murine colitis. Anat Rec 2010; 293: 849-857.

28 Chamoto K, Gibney BC, Lee GS, et al. CD34 ${ }^{+}$progenitor to endothelial cell transition in post-pneumonectomy angiogenesis. Am J Respir Cell Mol Biol 2012; 46: 283-289.

29 Chamoto K, Gibney BC, Lee GS, et al. Migration of $\mathrm{CD}_{11 \mathrm{~b}^{+}}$accessory cells during murine lung regeneration. Stem Cell Res 2013; 10: 267-277.

30 Renzoni EA, Walsh DA, Salmon M, et al. Interstitial vascularity in fibrosing alveolitis. Am J Respir Crit Care Med 2003; 167: 438-443.

31 Ackermann M, Houdek JP, Gibney BC, et al. Sprouting and intussusceptive angiogenesis in postpneumonectomy lung growth: mechanisms of alveolar neovascularization. Angiogenesis 2014; 17: 541-551. 

obliterans and alveolar fibroelastosis after lung and stem cell transplantation. I Pathol Clin Res 2016; 3: 17-28.

33 von der Thüsen JH, Hansell DM, Tominaga M, et al. Pleuroparenchymal fibroelastosis in patients with pulmonary disease secondary to bone marrow transplantation. Mod Pathol 2011; 24: 1633-1639.

34 Jacobo SM, Kazlauskas A. Insulin-like growth factor 1 (IGF-1) stabilizes nascent blood vessels. J Biol Chem 2015; 290: 6349-6360.

35 Ackermann M, Morse BA, Delventhal V, et al. Anti-VEGFR2 and anti-IGF-1R-adnectins inhibit Ewing's sarcoma A673-xenograft growth and normalize tumor vascular architecture. Angiogenesis 2012; 15: 685-695.

$36 \mathrm{Xi} \mathrm{Y}$, Kim T, Brumwell AN, et al. Local lung hypoxia determines epithelial fate decisions during alveolar regeneration. Nat Cell Biol 2017; 19: 904-914.

37 Varone F, Sgalla G, Iovene B, et al. Nintedanib for the treatment of idiopathic pulmonary fibrosis. Expert Opin Pharmacother 2018; 19: 167-175.

38 Jain RK. Normalization of tumor vasculature: an emerging concept in antiangiogenic therapy. Science 2005; 307: $58-62$.

39 Farkas L, Farkas D, Ask K, et al. VEGF ameliorates pulmonary hypertension through inhibition of endothelial apoptosis in experimental lung fibrosis in rats. J Clin Invest 2009; 119: 1298-1311.

40 Colombat M, Mal H, Groussard O, et al. Pulmonary vascular lesions in end-stage idiopathic pulmonary fibrosis: histopathologic study on lung explant specimens and correlations with pulmonary hemodynamics. Hum Pathol 2007; 38: 60-65.

41 Neubert L, Borchert P, Shin HO, et al. Comprehensive three-dimensional morphology of neoangiogenesis in pulmonary veno-occlusive disease and pulmonary capillary hemangiomatosis. J Pathol Clin Res 2019; 5: 108-114.

42 Ackermann M, Gaumann A, Mentzer SJ, et al. Plexiform vasculopathy in chronic thromboembolic pulmonary hypertension. Am J Respir Crit Care Med 2017; 196: e48-e51.

43 Wnuk M, Hlushchuk R, Tuffin G, et al. The effects of PTK787/ZK222584, an inhibitor of VEGFR and PDGFR $\beta$ pathways, on intussusceptive angiogenesis and glomerular recovery from Thy1.1 nephritis. Am J Pathol 2011; 178: 1899-1912.

44 Alvarez DF, Huang L, King JA, et al. Lung microvascular endothelium is enriched with progenitor cells that exhibit vasculogenic capacity. Am J Physiol Lung Cell Mol Physiol 2008; 294: L419-L430.

45 Misharin AV, Morales-Nebreda L, Reyfman PA, et al. Monocyte-derived alveolar macrophages drive lung fibrosis and persist in the lung over the life span. J Exp Med 2017; 214: 2387-2404.

46 Dimova I, Karthik S, Makanya A, et al. SDF-1/CXCR4 signalling is involved in blood vessel growth and remodelling by intussusception. J Cell Mol Med 2019; 23: 3916-3926. 